\title{
Entanglement-Induced Barren Plateaus
}

\author{
Carlos Ortiz Marrero,,${ }^{1, *}$ Mária Kieferová, ${ }^{2, \dagger}$ and Nathan Wiebe ${ }^{3,4, \$}$ \\ ${ }^{1}$ Data Sciences and Analytics Group, Pacific Northwest National Laboratory, Richland, Washington 99354, USA \\ ${ }^{2}$ Centre for Quantum Computation and Communication Technology, Centre for Quantum Software and \\ Information, University of Technology Sydney, New South Wales 2007, Australia \\ ${ }^{3}$ Department of Computer Science, University of Toronto, Ontario M5S 1A1, Canada \\ ${ }^{4}$ High Performance Computing Group, Pacific Northwest National Laboratory, Richland, Washington 99354, USA
}

(Received 15 February 2021; revised 21 July 2021; accepted 13 October 2021; published 25 October 2021)

\begin{abstract}
We argue that an excess in entanglement between the visible and hidden units in a quantum neural network can hinder learning. In particular, we show that quantum neural networks that satisfy a volume law in the entanglement entropy will give rise to models that are not suitable for learning with high probability. Using arguments from quantum thermodynamics, we then show that this volume law is typical and that there exists a barren plateau in the optimization landscape due to entanglement. More precisely, we show that for any bounded objective function on the visible layers, the Lipshitz constants of the expectation value of that objective function will scale inversely with the dimension of the hidden subsystem with high probability. We show how this can cause both gradient-descent and gradient-free methods to fail. We note that similar problems can happen with quantum Boltzmann machines, although stronger assumptions on the coupling between the hidden and/or visible subspaces are necessary. We highlight how pretraining such generative models may provide a way to navigate these barren plateaus.
\end{abstract}

DOI: 10.1103/PRXQuantum.2.040316

\section{INTRODUCTION}

In recent years, the prospects of quantum machine learning (QML) and quantum deep-neural networks have gained notoriety in the scientific community. QML builds on the success of traditional machine learning and the potential for quantum speed-up. The QML field has enjoyed increased attention for quantum algorithms for principal-component analysis [1], supportvector machines [2], kernel methods [3,4], and quantum neural networks (QNNs) [3,5-8] but experiences setbacks in the form of dequantization techniques [9-11].

A key part of a successful quantum machine-learning algorithm is an efficient training algorithm. In recent years, several barren-plateau results [12-16] have put limitations on the gradient-based training of QNNs. Quantum barren plateaus arise from random initialization of quantum machine models, which typically lead to configurations

\footnotetext{
*carlos.ortizmarrero@pnnl.gov

†maria.kieferova@uts.edu.au

$\ddagger$ nwiebe@cs.toronto.edu
}

Published by the American Physical Society under the terms of the Creative Commons Attribution 4.0 International license. Further distribution of this work must maintain attribution to the author(s) and the published article's title, journal citation, and DOI. where the objective function is not sensitive to small deviations of parameters. Hence, the gradients of the objective function with respect to the parameters are exponentially small. Our result complements the growing literature on barren plateaus in quantum computing. McClean et al. [12] showed that unitary quantum neural networks generically suffer from vanishing gradients exponentially in the number of qubits. This issue stems from the concentration of measure $[17,18]$ and has subsequently been demonstrated for other QNNs [13,14]. Another type of a barren plateau emerges from hardware noise in the system [15]. The key observation that we put forward in this work is that barren plateaus can occur because of an excess of entanglement in deep quantum models.

In this paper, we prove that entanglement between visible and hidden units hinders the learning process. The inclusion of hidden units is essential in traditional machine learning. Without them, the expressive power of neural networks would be severely limited and deep learning all but impossible. In spite of this, there has been very little attention paid to the effect of hidden units on the training of QNNs. Surely, the expressive power of hidden units would translate to the quantum world. Numerical experiments seem to contradict this intuition. A small-scale numerical study [19] has shown that the inclusion of hidden units in quantum Boltzmann machines does not lead to a higher quality of reproduction. While this can be explained as 
being due to the small size of the QNN and simple data, in our work we show that quantum Boltzmann machines do not benefit from a large number of hidden units.

We build intuition from exploring the statistical relationship between a random state and maximally entangled states in a bipartite quantum system. This intuition has been well explored within the thermalization community, which examines how statistical-mechanical ensembles (such as the microcanonical ensemble or the canonical ensemble) emerge for closed quantum systems through entanglement between the system of interest and a reservoir, making the resulting density matrices practically indistinguishable from the predictions of the corresponding ensemble when $D_{\text {system }} \ll D_{\text {reservoir }}$. In particular, we build upon a classic thermalization result [20] that shows that for a random initial state, the state on the visible units is with high probability exponentially close to a maximally mixed state. However, if the state is chosen from a $k$ design, then its distance to a maximally mixed state scales as $\left(D_{v}^{3} / D_{h}\right)^{k}$ which is seen in Theorem 5.3 of Ref. [21]. We show that it is very difficult to escape from this state because the gradients will be exponentially small. As such, for a wide array of QNNs, randomness and entanglement hinder the training.

This surplus of entanglement to some extent defeats the purpose of deep learning by causing information to be nonlocally stored in the correlations between the layers rather than in the layers themselves. Since the objective function has, by definition, support only on visible units, only these qubits will be measured. As is customary in deep learning, the state of hidden units is ignored, which can be seen as tracing it out. Thus, when considering only the visible subsystem, we find that the resulting state is close to the maximally mixed state. Indeed, we show that such situations are generic as well and that gradient-descent methods are unlikely to allow the user to escape from such a plateau at low cost. This observation holds for both "feed-forward" QNNs as well as Boltzmann machines and suggests that if quantum effects are to be used to improve classical models, then they must be used surgically. Furthermore, our work establishes a link between the thermalization literature and quantum machine learning that has been hitherto absent from the literature [22,23].

We focus on two types of QNNs depicted in Fig. 1, feed-forward unitary quantum neural networks inspired by the quantum approximate-optimization algorithm (QAOA) and quantum Boltzmann machines [8,19,24].

Quantum Boltzmann machines can also be trained generatively [19], meaning that rather than optimizing a training objective function that is a linear function of the density operator, such as $\operatorname{Tr}\left[O_{\mathrm{Obj}} \operatorname{Tr}_{h}\left(e^{-H} / Z\right)\right]$, we aim to optimize a nonlinear function of the density operator such as the quantum relative entropy, i.e., $S\left[\rho_{\text {train }} \| \rho(\theta)\right]=\operatorname{Tr}\left\{\rho_{\text {train }} \log \left(\rho_{\text {train }}\right)-\rho_{\text {train }} \log [\rho(\theta)]\right\}$, by generating a quantum state $\rho(\theta)$ using the Boltzmann machine that optimizes this divergence with the training density operator.

A key assumption of our results is that an untrained quantum neural network produces outcomes that are close to being random. Specifically, we assume that the initial circuit for the unitary model can be seen as sampled from an unitary 2-design. This assumption is well justified for quantum circuits with a depth that grows polynomially in the number of input qubits and that are generated by random sequences of universal gates [25-27]. A random circuit consisting of $l$ layers of alternating operators in the $Z$ or $X$ basis can also approximate a 2-design to accuracy $\Theta\left(D^{-l}\right)$, where $D$ here is the dimension of the Hilbert space [28]. Lastly, there are exact and approximate 2-designs that can be implemented by logarithmic-depth quantum circuits $[29,30]$; however, it is unclear how likely one would be to accidentally initialize a unitary quantum neural network to such a construction. In the case of quantum Boltzmann machines, we make assumptions about the eigendecomposition of the initial Hamiltonian that are stated in Theorem 4. We introduce a parameter $\Gamma$ that characterizes the bound between energy levels of the Hamiltonian and figures in the bound on eigenvalues. We further assume that the eigenvectors of the Hamiltonian are typical of the columns of a random matrix drawn from a unitary 2-design. Such distributions are commonplace for Hamiltonians that describe complex quantum systems [31].

\section{THE IMPACT OF ENTANGLEMENT ON DEEP MODELS}

The central question of our work is to understand how the entanglement in the neural network affects the visible units. Instead of providing speed-up, entanglement between visible and hidden units causes thermalization on the visible subsystem. Thus, the inclusion of entanglement between the hidden and visible layers of a QNN can, unless carefully controlled, be harmful to the neural network model.

The relationship between the representational power of a neural network and the degree of entanglement between the visible and hidden systems has been discussed in Ref. [32]; however, here we reexamine this question and arrive at a different conclusion. Specifically, we conclude that large amounts of entanglement (as quantified by a volume law) can be catastrophic for the model; whereas an area-law scaling for the entanglement entropy between the hidden and visible can often be tolerated.

To see this, we need to make a few formal definitions. Let $\mathcal{S} \subset \mathbb{C}^{D_{v} D_{h} \times D_{v} D_{h}}$ be a family of parametrized density operators, where $D_{v}=2^{n_{v}}$ is the dimension of the $n_{v^{-}}$ qubit visible subspace and $D_{h}=2^{n_{h}}$ is the dimension of the hidden subspace. For each $\rho \in \mathcal{S}$, the qubits can be uniquely assigned to the vertices of a graph $\mathcal{G}$ on a vertex set $\mathcal{V}_{h} \cup \mathcal{V}_{v}$, where $\mathcal{V}_{v}$ consists of $\log _{2}\left(D_{v}\right)=n_{v}$ and 


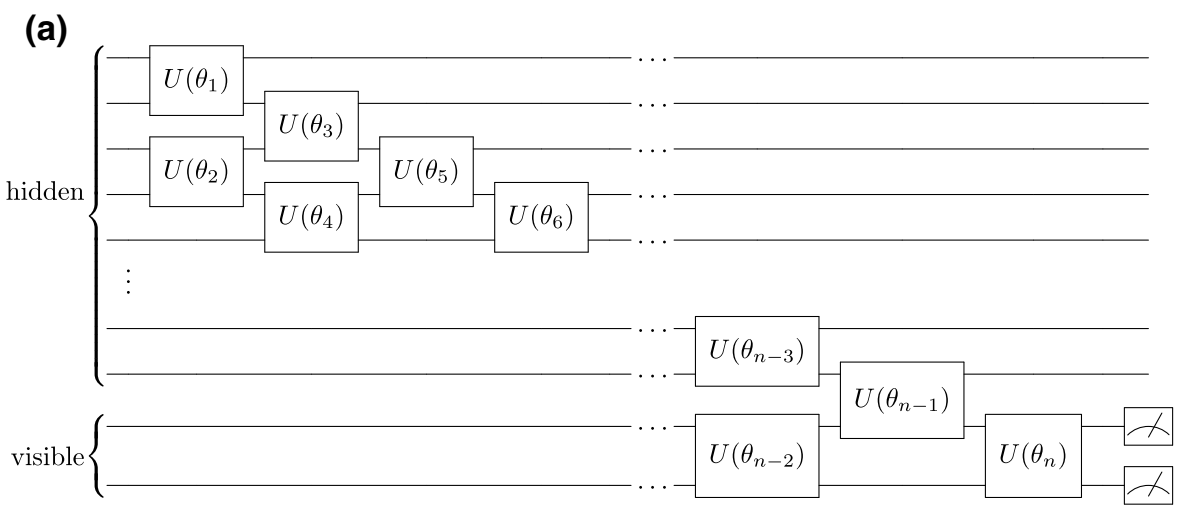

(b)

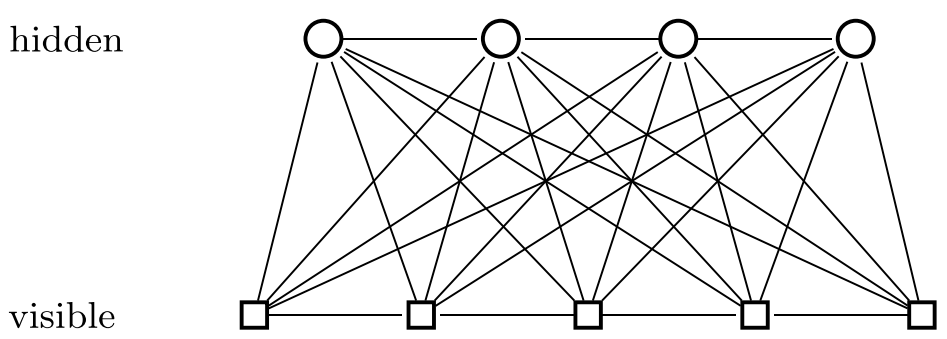

FIG. 1. Examples of QNNs. (a) A quantum unitary network characterized by a circuit with parametrized unitaries $U_{j}=e^{-i \theta_{j} H_{j}}$, where the $\theta_{j}$ are the parameters that we aim to learn and the $H_{j}$ are Hamiltonians that specify the QNN. The output is then $U\left(\theta_{1}, \ldots, \theta_{n}\right)\left|\psi_{0}\right\rangle$, where $\left|\psi_{0}\right\rangle$ can be taken to be $|0 \ldots 0\rangle$ for generative learning. In this model, visible units correspond to the qubits on which we evaluate the objective function, in this case the last two registers. The remaining qubits are called hidden units. (b) Quantum Boltzmann machines (QBMs) defined on a graph. Each edge and each vertex correspond to a weight on a local Hamiltonian corresponding to the pair of qubits or a single qubit. The top layer of units (circles) corresponds to visible units and the bottom layer (rectangles) are hidden units. QBMs model data as a thermal state $e^{-H(\theta)} / Z(\theta):=e^{-\sum_{i} \theta_{i} H_{i}} / \operatorname{Tr}\left(e^{-\sum_{i} \theta_{i} H_{i}}\right)$. Without loss of generality, we take $\operatorname{Tr}(H)=0$ for all quantum Boltzmann machines. The aim when training a quantum Boltzmann machine is to learn a vector $\theta$ such that for a training objective function given by $O_{\text {obj }}$ that acts on the visible subsystem, we maximize $\operatorname{Tr}\left(O_{\mathrm{obj}} \operatorname{Tr}_{h}\left[e^{-H(\theta)} / Z(\theta)\right]\right.$.

$\mathcal{V}_{h}$ of $\log _{2}\left(D_{h}\right)=n_{h}$ qubits. We then define $n_{v}^{(j)}$ to be the number of vertices in $\mathcal{V}_{v}$ that are at least graph distance $j$ away from the vertices in $\mathcal{V}_{h}$ and we define $n_{h}^{(j)}$ to be the analogous number for the vertices in $\mathcal{V}_{h}$ (see Fig. 2). We then say that $\mathcal{S}$ satisfies an area law if for all $\rho \in \mathcal{S}$, $S\left[\operatorname{Tr}_{h}(\rho)\right] \in \Theta\left(n_{v}^{(1)}\right)$; similarly, we say that $\mathcal{S}$ satisfies a volume law if $\left|S\left[\operatorname{Tr}_{h}(\rho)\right]-\log \left(D_{v}\right)\right| \in \Theta\left(D_{v} / D_{h}\right)$, where $S$ is the von Neumann entropy. Note that here we specifically choose a slightly stronger definition of a volume-law scaling than is used in some other settings. In our context, the leading-order constant factor is important to bound the remainder term for a finite value of $D_{h}$. With these definitions in place, we can concisely claim our result.

Proposition 1. Let $\mathcal{S}$ be a family of density operators with visible dimension $D_{v}$ and hidden dimension $D_{h} \geq D_{v}$, with $n_{v}^{(1)}$ and $n_{h}^{(1)}$ qubits in the first visible and hidden layers, respectively. We then have that for any operator on the visible subsystem $O_{o b j}$ and $\rho \in \mathcal{S} \subset \mathbb{C}^{D_{v} D_{h} \times D_{v} D_{h}}$,

$$
\left|\operatorname{Tr}\left\{\left(O_{o b j} \otimes I_{h}\right)\left[\rho-I /\left(D_{v} D_{h}\right)\right]\right\}\right|
$$

is in $O\left(\left\|O_{O b j}\right\|_{\infty} \sqrt{\log \left(D_{v}\right)-n_{v}^{(1)}}\right)$ if $\mathcal{S}$ satisfies an area law and is in $O\left(\left\|O_{O b j}\right\|_{\infty} \sqrt{\left(D_{v} / D_{h}\right)}\right)$ if $\mathcal{S}$ satisfies a volume law, where $\|\cdot\|_{\infty}$ is the spectral norm of an operator.

Proof. The proof follows from standard inequalities for the quantum relative entropy:

$$
\begin{aligned}
\frac{1}{2}\left\|\operatorname{Tr}_{h}(\rho)-I_{v} / D_{v}\right\|_{1}^{2} & \leq S\left[\operatorname{Tr}_{h}(\rho) \| I_{v} / D_{v}\right] \\
& =-S\left[\operatorname{Tr}_{h}(\rho)\right]+\log \left(D_{v}\right) .
\end{aligned}
$$

Then, from the von Neumann trace inequality, we have

$$
\begin{aligned}
& \left|\operatorname{Tr}\left[\left(O_{\mathrm{Obj}} \otimes I_{h}\right)\left(\rho-I_{v} / D\right)\right]\right| \leq\left\|O_{\mathrm{Obj}}\right\|_{\infty}\left\|\operatorname{Tr}_{h}(\rho)-I_{v} / D_{v}\right\|_{1} \\
& \quad \leq\left\|O_{\mathrm{Obj}}\right\|_{\infty} \sqrt{2\left\{\log \left(D_{v}\right)-S\left[\operatorname{Tr}_{h}(\rho)\right]\right\}}
\end{aligned}
$$

If $\rho$ satisfies an area-law scaling, then $S\left[\operatorname{Tr}_{h}(\rho)\right] \in \Theta\left(n_{v}^{(1)}\right)$, from which the claimed result for the area-law scaling 


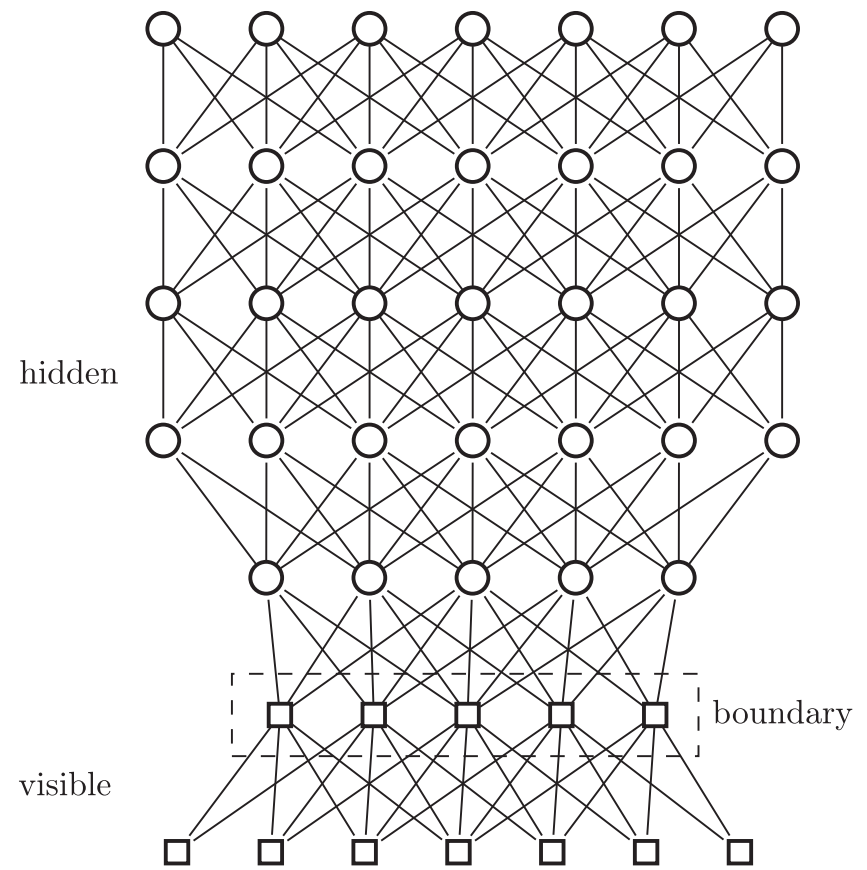

FIG. 2. For an area law, the entanglement entropy scales as the number of qubits on the boundary (in the dashed rectangle).

immediately follows. If, instead, we assume that $\rho$ obeys a volume law, then $\left|S\left[\operatorname{Tr}_{h}(\rho)\right]-\log \left(D_{v}\right)\right| \in \Theta\left(D_{v} / D_{h}\right)=$ $\Theta\left(2^{n_{v}-n_{h}}\right)$.

This shows that if our quantum neural network outputs states that satisfy a volume law, then asymptotically the predictions of the neural network would be no better than random guessing. In contrast, quantum neural networks will not necessarily observe this problem if the entanglement entropy is characteristic of an area-law scaling unless the number of hidden units in the first layer becomes much larger than the number of visible units. We therefore see that uncontrolled entanglement, such as that yielded by volume laws, can be catastrophic for a quantum variant of deep-neural networks that requires a number of hidden units much larger than the visible units. Instead, limited entanglement yielded by area laws may be more desirable. This means that when designing neural networks, it is vital to aim for sub-volume-law scaling. However, such states often have concise representations using matrix-product states [33] and so might be no more performant than classical neural networks. Nonetheless, we show in Sec. III that such sub-volume-law scalings are not typical and that almost all quantum neural networks within the ensembles that we consider obey volume-law scalings.

\section{TYPICALITY OF VOLUME-LAW SCALING}

While area laws occur for certain systems [34,35], such as ground states of gapped translationally invariant Hamiltonians on lattices, we expect volume-law scalings to be much more common. This intuition can be made rigorous by making appropriate assumptions about the interactions between the visible and hidden layers in the model. In particular, we assume that the quantum states on the joint system of the QNN approximate a Haar random state. In practice, this assumption is too strong, as Haar random states typically require exponentially long quantum circuits to generate them. Instead, we focus on ensembles generated by unitary 2-designs.

Proposition 2. Let $U \in \mathbb{C}^{D_{v} D_{h} \times D_{v} D_{h}}$ be drawn from a unitary 2-design and let $H=U^{\dagger} S U$ for some diagonal matrix $S \in \mathbb{C}^{D \times D}$, where $D=D_{v} D_{h}$. If either $\rho=$ $U|0\rangle\langle 0| U^{\dagger}$ (unitary network) or $\rho=e^{-H} / \operatorname{Tr}\left(e^{-H}\right)$ (Boltzmann machine), then for any bounded operator $O_{o b j} \in$ $\mathbb{C}^{D_{v} \times D_{v}}$ acting on the visible subspace, we have that

$$
\left|\operatorname{Tr}\left[\left(O_{o b j} \otimes I_{h}\right)(\rho-I / D)\right]\right| \in O\left(\left\|O_{o b j}\right\|_{\infty} \sqrt{\frac{D_{v}}{D_{h}}}\right)
$$

with high probability over $U$.

Proof. Let us first examine the case of $\rho=U^{\dagger}|0\rangle\langle 0| U$. We have that if we take the expectation value over $U$ drawn from a 2-design, then

$$
\begin{aligned}
& \left|\mathbb{E}\left(\operatorname{Tr}\left[\left(O_{\text {obj }} \otimes I_{h}\right)\left(U^{\dagger}|0\rangle\langle 0| U-I / D\right)\right]\right)\right| \\
& \quad \leq\left\|O_{\text {obj }}\right\|_{\infty} \mathbb{E}\left\|\operatorname{Tr}_{h}\left(U^{\dagger}|0\rangle\langle 0| U-I / D\right)\right\|_{1} \\
& \quad \leq\left\|O_{\text {obj }}\right\|_{\infty} \sqrt{D_{v} \mathbb{E}\left\|\operatorname{Tr}_{h}\left(U^{\dagger}|0\rangle\langle 0| U-I / D\right)\right\|_{2}^{2}} .
\end{aligned}
$$

Since the partial trace of a density operator is a density operator, it follows that the argument is positive definite and in turn that the result can be written as

$$
\left\|O_{\text {obj }}\right\|_{\infty} \sqrt{\mathbb{E}\left(\operatorname{Tr}[(\rho-I / D) \otimes(\rho-I / D)]\left(F_{v v^{\prime}} \otimes I\right)\right)},
$$

where $F_{v v^{\prime}}$ is the flip or swap operator that swaps the two visible subsystems.

Since the result is quadratic in the probability distribution, we have, from the definition of a unitary 2-design, that

$$
\begin{aligned}
& \mathbb{E}(\operatorname{Tr}[(\rho-I / D) \otimes(\rho-I / D)]) \\
& \quad=\mathbb{E}_{\text {Haar }}(\operatorname{Tr}[(\rho-I / D) \otimes(\rho-I / D)]),
\end{aligned}
$$

where $\mathbb{E}_{\text {Haar }}$ is the Haar expectation value. The result then follows immediately from invoking Theorem 2 from the 
result of Popescu et al. [20], that

$$
\begin{aligned}
& \left\|O_{\text {obj }}\right\|_{\infty} \sqrt{\mathbb{E}\left(\operatorname{Tr}[(\rho-I / D) \otimes(\rho-I / D)]\left(F_{v v^{\prime}} \otimes I\right)\right)} \\
& \quad \in O\left(\left\|O_{\text {obj }}\right\|_{\infty} \sqrt{\frac{D_{v}}{D_{h}}}\right) .
\end{aligned}
$$

Next, let us assume that $\rho=e^{-H} / \operatorname{Tr}\left(e^{-H}\right)$. We have, from the definition of $H$ and the previous result, that for any eigenvector $|j\rangle$ of $H$,

$$
\left|\operatorname{Tr}\left[\left(O_{\mathrm{obj}} \otimes I_{h}\right)(|j\rangle\langle j|-I / D)\right]\right| \in O\left(\left\|O_{\mathrm{obj}}\right\|_{\infty} \sqrt{\frac{D_{v}}{D_{h}}}\right) .
$$

Since $\rho=\sum_{j}|j\rangle\langle j| e^{-\langle j|H| j\rangle} / \operatorname{Tr}\left(e^{-H}\right):=\sum_{j} \mathbb{E}_{H}(|j\rangle\langle j|)$, the required result immediately follows by interchanging the order of the expectation values over the mixed state and over the unitary 2-design. These results also hold with high probability as a consequence of Markov's inequality.

This shows that for both the Boltzmann machine as well as unitary quantum networks, any observable measured on the visible layers will be indistinguishable, in expectation, to the maximally mixed state with high probability. In other words, the presence of entanglement actually weakens these models as the dimension of the hidden subsystem grows relative to the visible subsystem. For deep networks, we anticipate that there will be many more hidden neurons than visible neurons and hence that, generically, entanglement is a bane, not a boon, for deep QNNs.

There are a number of caveats to this analysis. First, we assume that the states in question are typical of a unitary 2-design. This assumption may not be appropriate if a structured ansatz is used or if the used circuits are shallow. The next assumption is that the observable is supported on the visible system only. The final, potential, caveat is that gradient-based optimizers may allow us to train our way out of these typical points and thereby find a way to productively leverage quantum effects. While the first two caveats do speak to ways to escape this apparent no-go result, the ubiquity of "entanglement-induced barren plateaus" will make the third option fail with high probability.

\section{ENTANGLEMENT-INDUCED BARREN PLATEAUS}

Our arguments for why gradient descent will fail to improve the quality of a training objective function due to entanglement between the visible and hidden layers follows from reasoning similar to that employed in Proposition 2 . However, the specific arguments require slightly more nuanced assumptions, since we need to worry about how perturbations to the model parameters impact the resulting state. Such assumptions are also made, for example, in the original McClean et al. work that identified barren plateaus for unitary networks [12]. Further, while we are able to directly employ existing results from the literature of thermalization to prove Proposition 2, the necessary conditions do not hold for the gradients operator. We state the main results below and provide an explicit proof in Appendices A and B.

\section{A. Plateaus for unitary networks}

Here, we prove one of the main results of our paper, a barren plateau for a unitary neural network. We consider the case of unitary networks of the form

$$
U\left(\theta_{1}, \ldots, \theta_{n}\right):=e^{-i H_{n} \theta_{n}} \ldots e^{-i H_{1} \theta_{1}},
$$

as depicted in Fig. 1(a). We consider the case where one of the parameters is shifted by a constant amount $\delta_{k}$ and argue about the maximum possible shift in the expectation of an observable that is supported only on the visible subsystem. Formally, we give a bound on the Lipshitz constant $\Lambda$ of the expectation value of $O_{\mathrm{obj}}$ such that

$$
\Lambda \geq \frac{\left|\operatorname{Tr}_{v}\left\{O_{\mathrm{obj}} \operatorname{Tr}_{h}\left[\rho(\theta)-\rho\left(\theta+\delta_{k}\right)\right]\right\}\right|}{\left|\delta_{k}\right|}
$$

for all deviations $\delta_{k}$ from the model parameters $\theta$.

A major challenge to analyzing what happens when shifting parameters of a unitary network is that such networks are so complicated that the impact of this perturbation is difficult to measure. An example of such an effect can be seen in the Loschmidt echo, which shows exponential sensitivity to perturbations in the parameters of complex quantum dynamics [31,36]. Our solution, similar to that taken in Ref. [12], is to assume that the dynamics scrambles the states so much that almost all subsequences of the product $\prod_{j=1}^{k} e^{-i H_{j} \theta_{j}}$ form a unitary 2-design. We then see that the value of the objective function is Lipshitz continuous with a constant that scales inversely with the hidden dimension $D_{h}=2^{n_{h}}$. This shows that the plateau exists both for gradient-descent as well as gradient-free methods [37]. A formal statement of this intuition and the result are given below.

Theorem 3 (Gradient in Unitary Networks.). Assume that $\rho(\theta)$ is drawn from a unitary 2-design, where $\rho(\theta)$ is generated through a unitary ansatz of the form

$$
\rho(\theta)=\prod_{j=1}^{N} e^{-i H_{j} \theta_{j}}|0\rangle\langle 0| \prod_{j=N}^{1} e^{i H_{j} \theta_{j}}
$$

that acts on a Hilbert space that is the product of a hidden and visible space of dimensions $D_{h}$ and $D_{v}$, respectively. 
Further, define that $H_{k}(\theta)=\prod_{j=1}^{k} e^{-i H_{j} \theta_{j}} H_{k} \prod_{j=k}^{1} e^{i H_{j} \theta_{j}}$ for each $k$ obeys $\mathbb{E}\left[H_{k}(\theta) \rho(\theta)\right]=\mathbb{E}\left[H_{k}(\theta)\right] \mathbb{E}[\rho(\theta)]$. We then have that

$$
\mathbb{E}\left(\left|\operatorname{Tr}\left\{O_{o b j} \operatorname{Tr}_{h}[\rho(\theta)]\right\}\right|\right)
$$

is a Lipshitz continuous function of $\theta$ with constant $\Lambda$, obeying

$$
\Lambda \in O\left(\left\|O_{o b j}\right\|_{\infty}\left\|H_{k}\right\|_{\infty} \sqrt{\frac{D_{v}}{D_{h}}}\right) .
$$

The proof of the theorem follows by using the unitary invariance of the trace norm and Hadamard's lemma to rewrite the difference between the perturbed exponential and the original exponential as a commutator series of $H_{k}(\theta)$ and $\rho(\theta)$. We then use the triangle inequality, Cauchy-Schwarz inequality, and the independence assumption made above to arrive at the result. An explicit proof is given in Appendix A. A similar result can be also obtained from Levy's lemma under a much stronger assumption, Haar randomness. We provide this result in Sec. V.

\section{B. Plateaus for Boltzmann machines}

Next, we turn our attention to Boltzmann machines. We show that parametrized Hamiltonians drawn from a unitary ensemble also experience an entanglement-induced barren plateau. The nature of this plateau, however, differs from that of the plateau of the unitary network in that it occurs under reasonable assumptions if $\operatorname{Tr}\left(h_{h}\right)^{2} / \operatorname{Tr}\left(h_{h}^{2}\right) \in o\left(D_{h}\right)$, as we see below.

Theorem 4 (Gradient for Boltzmann Machines.). Assume that $H \in \mathbb{C}^{D \times D}$ is a random Hermitian matrix drawn from an ensemble in the following manner: a diagonal matrix with eigenvalues $E_{j} \in \mathbb{R}$ is chosen according to a probability $\operatorname{Pr}\left(E_{j}\right)$ such that $\max _{k} \mathbb{E}\left\{1 / D^{2}\left[\sum_{j \neq k}\left(E_{j}-\right.\right.\right.$ $\left.\left.\left.E_{k}\right)^{-1}\right]^{2}\right\} \leq \Gamma^{2}$ and is then conjugated with a unitary drawn from a distribution that is a unitary 2-design. Let $H_{k}=$ $h_{v} \otimes h_{h} \in \mathbb{C}^{D \times D}$ where $h_{v}, h_{h}$ are fixed Hermitians acting on the visible and hidden subspace, respectively, and

$$
\rho\left(\theta_{k}\right):=e^{-\left(H+\theta_{k} H_{k}\right)} / \operatorname{Tr}\left(e^{-\left(H+\theta_{k} H_{k}\right)}\right) .
$$

Finally, let $O_{o b j} \in \mathbb{C}^{D_{v} \times D_{v}}$ be a Hermitian matrix. Then

$$
\kappa:=\operatorname{Tr}\left\{\left(O_{o b j} \otimes I_{h}\right)\left[\rho\left(\theta_{k}\right)\right]\right\}
$$

is a differentiable function that obeys

$$
\left|\frac{\partial \kappa}{\partial \theta_{k}}\right|_{\theta_{k}=0} \in O\left(\left\|O_{o b j}\right\|_{\infty} \Gamma\left\|H_{k}\right\|_{\infty} \sqrt{\left.\frac{D_{v}}{D_{h}}\left(\frac{\operatorname{Tr}\left(h_{h}\right)^{2}}{D_{v} \operatorname{Tr}\left(h_{h}^{2}\right)}+1\right)\right)},\right.
$$

with high probability over the ensemble.
The proof of Theorem 4 can be found in Appendix B. The sketch of the proof is relatively simple. We use the assumption that the eigenvectors are taken to be columns of matrices drawn from a unitary 2-design and then use perturbation theory to argue about the perturbed $H$. The use of perturbation theory introduces the parameter $\Gamma$ that characterizes the inverse minimal gap. We then take the partial trace of the resulting perturbed eigenvectors to show that if the reduced density matrix over the hidden units of the perturbation Hamiltonian $H_{k}$ has zero trace, then the partial trace over the hidden layers of each eigenvector remains the maximally mixed state as per Proposition 2. This partial-trace assumption is needed because if bias terms are added to the hidden units then one can disentangle them from the visible units in the ground state through the perturbation. While such a perturbation may save the predictive power of the Boltzmann machine, it effectively eliminates the hidden layers, causing the model to revert to a shallow one. With these observations, the results then follow from the use of standard inequalities and the Haar expectation value of random states given, for example, in Ref. [38]. The result holds with high probability as a consequence of Markov's inequality.

In particular, we find that the gradient of the objective function with respect to terms that nontrivially act on the hidden layers is exponentially small in the number of hidden qubits since, without loss of generality, we may take $\operatorname{Tr}\left(h_{h}\right)=0$ for all such terms. In contrast, the gradient with respect to the visible Hamiltonian coefficients need not be exponentially small in the number of hidden qubits. Indeed, if we have a $k$-local random Hamiltonian where each Hamiltonian coefficient is chosen independently from a distribution that is independent of $D$, then $\Gamma \in O\left[\log (D)^{1-k}\right]$; thus, for any $k \geq 2$, the gradient may only be polynomially small.

A side effect of these observations is that they explain, in part, the observations in Ref. [19] that the number of hidden units included in the model does not increase the performance of quantum Boltzmann machines. This can now be understood from the fact that the Gibbs states for typical Hamiltonians generate thermal states that are close to the maximally mixed state. Thus, the inclusion of hidden units will not typically be expected to increase the performance of quantum Boltzmann machines.

\section{HAAR RANDOM UNITARIES}

In the previous sections, we assume that the eigenbasis the neural networks scramble at least as effectively as a unitary 2-design. However, if we assume that in the case of the unitary networks the gate sequence is Haar random or, in the case of the Boltzmann machine, that the basis is Haar random, then the type of concentration that we see can be radically improved. Specifically, Levy's lemma [20] can be used in place of Markov's inequality to show that 
the vast majority of randomly selected networks will have vanishing gradients. In particular, we have the following.

Lemma 5 (Levy.). Given a function $f: U(D) \mapsto \mathbb{R}$ that is Lipshitz with constant $\eta$ with expectation value over the Haar measure $\mathbb{E}(f)$, it follows that there exists $C \in \Theta(1)$ such that, for any $U$ sampled from the Haar measure on $U(D)$,

$$
\operatorname{Prob}[|f(U)-\mathbb{E}(f)| \geq \epsilon] \leq 2 \exp \left(\frac{-C(D+1) \epsilon^{2}}{\eta^{2}}\right),
$$

In the context of training QNNs, the function $f$ in Levy's lemma denotes the objective function evaluated on visible units $\operatorname{Tr}\left\{O_{\mathrm{obj}} \operatorname{Tr}_{h}[\rho(\theta)]\right\}$. This result ends up allowing us to use an even-tighter-concentration result for the systems than is possible using Markov's inequality, because it shows that a large deviation from the Haar expectation is exponentially small. This further means that a substantial deviation from the results stated above is in fact exponentially smaller than would be expected if we only had a 2-design condition. If unitary $k$-designs are used in place of 2-designs, then it should be noted that it is possible to interpolate between these two results [21], however, the bounds that arise from using this result under the assumption that we only have a 2-design are not superior to our Markov-based analysis.

\section{NUMERICAL RESULTS}

We run a series of numerical experiments, summarized in Figs. 3 and 4, to demonstrate that our asymptotic results apply to small-sized quantum networks.

We construct our ansatz drawing terms from a random 2-local Hamiltionian model on $n$-qubits. Let $\sigma_{a}^{j}=I^{\otimes j-1} \otimes$ $\sigma_{a} \otimes I^{\otimes n-j}$ for $a \in\{x, y, z\}$ and define

$$
\hat{H}=\sum_{i} \sum_{a} J_{a}^{i} \sigma_{a}^{i}+\sum_{i<j} \sum_{a} \sum_{b} J_{a, b}^{i, j} \sigma_{a}^{i} \sigma_{b}^{j},
$$

where we refer to $J_{a}^{i}$ as the on-site coefficients and $J_{a, b}^{i, j}$ as the off-site coefficients of our model. For the unitary model, we exhaustively sample from the individual terms in Eq. (11) to construct the individual unitaries. For the Boltzmann model, we use $\hat{H}$ as our Hamiltonian.

In Figs. 3(a) and 4, we compare the trace-distance scaling of the maximally mixed state and three models: the Gaussian unitary ensemble model, the unitary QNN, and the quantum Boltzmann machine. In Fig. 3(a), we see that for an increasing number of hidden units, these models will produce states close to the maximally mixed state. This result can be understood in the context of Sec. III. Figure 4 highlights this effect on the data histograms: as we increase the number of hidden units, we see the trace distance concentrating around zero.

In Fig. 3(b), we measure how the gradients of the unitary QNN change in relation to the number of hidden units. We observe an overall decrease in $\infty$ norm of the gradient vector as we increase the hidden units of our model. We generate a fixed thermal state $e^{-\hat{H}} / Z$ with on-site coefficients drawn from a normal distribution with mean 0 and variance 0.01 , i.e., $\mathcal{N}(0,0.01)$ and $\mathcal{N}(0,1)$, for the off-site coefficients. We then proceed to estimate the gradient vector of the fidelity, $F$, between our model and this fixed state using finite differences. We generate 1000 instances of our unitary model by initializing all the coefficients with samples from $\mathcal{N}(0,1)$. The figure shows an average decrease in the $\infty$ norm of the gradient vector on a semilog scale. We also calculate the exponential rate of (a)

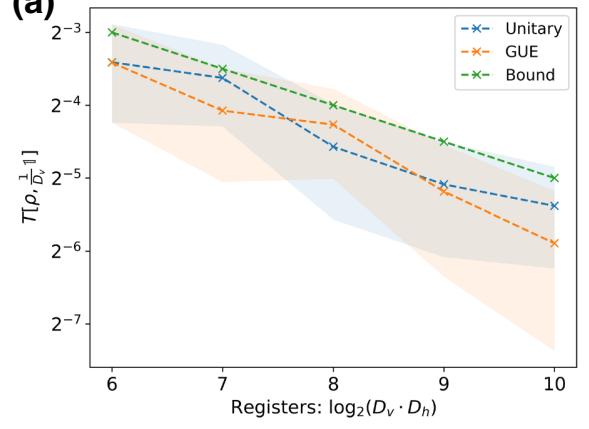

(b)

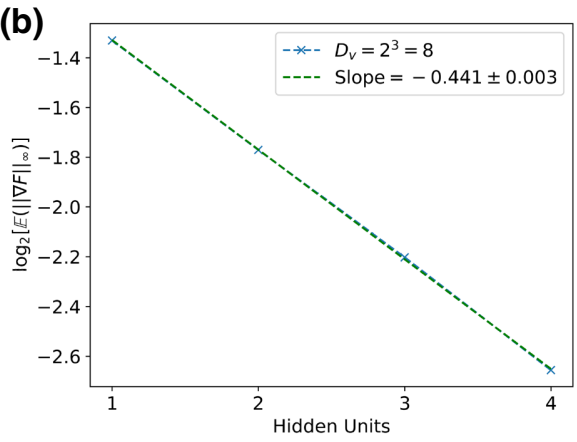

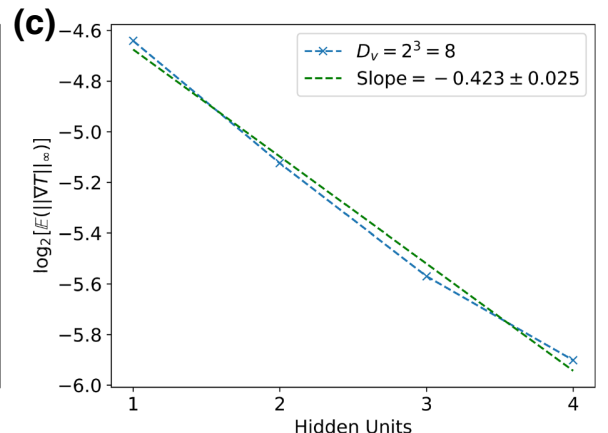

FIG. 3. (a) A log-log plot showing the trace-distance data in relation to our bound. The blue and orange marked values correspond to the estimated maximum peaks of the data histograms of 1000 model instances and the width of the shaded area corresponds to two standard deviations for a fix $D_{v}=2^{1}=2$. The green marked values are our bound results, i.e., $\mathbb{E}[T(\rho, I / D)] \leq 1 / 2 \sqrt{D_{v} / D_{h}}$. (b),(c) Semilog plots highlighting the decay in the expected value of the $\infty$ norm of the gradient vector over an ensemble of initialized models. The dashed blue line represents the average of 1000 model instances. The dash green line represents the best fit obtained from least squares with the standard error of the estimated slope under the assumption of residual normality: (b) gradient estimates for the unitary model; (c) gradient estimates for the normalized quantum Boltzmann machine. 
(a)

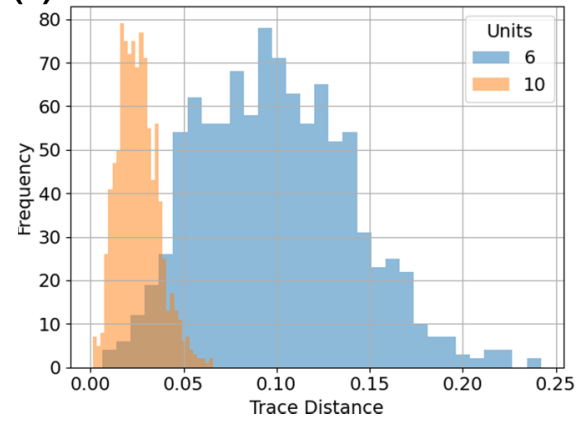

(b)

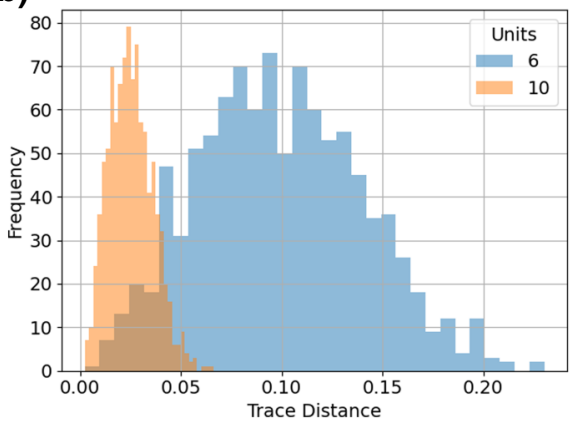

(c)

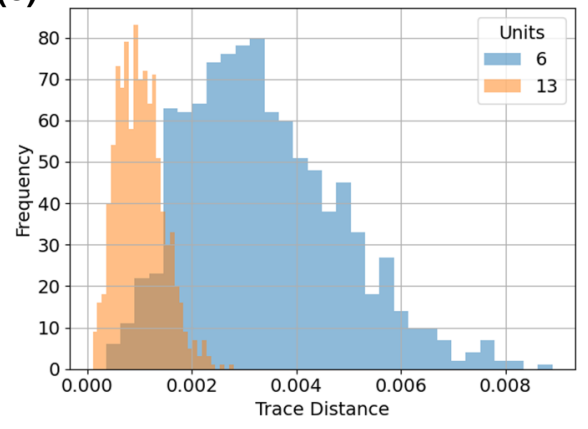

FIG. 4. Computation of the trace distance between the reduced density matrices of our models and the maximally mixed state for 1000 instances. The models considered have only one visible unit, i.e., $D_{v}=2^{1}=2$. (a) The empirical trace-distance distribution of a real-time evolution $\left(e^{-i H t}\right.$, for $\left.t=10\right)$ of Hamiltonians drawn from the Gaussian unitary ensemble (GUE). (b) The empirical tracedistance distribution of the unitary model. All coefficients are drawn from a uniform distribution over $[0,1)$. (c) The empirical tracedistance distribution of the quantum Boltzmann machine. The on-site coefficients, $J_{a}^{i}$, are drawn $\mathcal{N}(0,0.01)$. The off-site coefficients, $J_{a, b}^{i, j}$, are drawn from $\mathcal{N}(0,1)$. Moreover, the Hamiltonian is normalized by its operator norm.

decay using least-squares fitting. We see the same overall decay as we increase the number of hidden units that is predicted in Theorem 3, despite the fact that the fidelity is not an objective function in the form described by the theorem. We also observe an overall decay in the variance of the $\infty$ norm of the gradient vector as we increase the number of hidden units. We include these results in Appendix 5.

The Boltzmann-machine results are summarized in Fig. 3(c). In order to observe gradient decay in our experiments, we need to amplify the effect of the off-site terms in relation to the on-site terms to encourage a volumelaw scaling. The emergence of these volume laws can be understood from perturbation theory, since the leadingorder shift in an eigenvector $|n\rangle$ with eigenvalue $E_{n}$ proportional to $\sum_{j \neq n}|j\rangle\left\langle j\left|H_{k}\right| n\right\rangle /\left(E_{n}-E_{j}\right)$. This shows that if we take $|n\rangle$ to be an eigenstate of the one-body terms in the Hamiltonian, then the entanglement generated by $H_{k}$ is suppressed by the energy gaps between these states. We, therefore, choose these magnitudes to be small, so that significant entanglement can be introduced in the eigenstates despite the small values of $D$, which can be explored on a classical computer.

In Fig. 3(c), we estimate the $\infty$ norm of the gradient vector of the trace distance, $T$, between our model and its perturbation along each direction in the space of parameters using finite differences (see Theorem 4). The on-site coefficients are drawn from $\mathcal{N}(0,0.01)$ and the offsite coefficients from $\mathcal{N}(0,1)$. Moreover, we normalize the Hamiltonian by its operator norm. Although the results of Theorem 4 do not apply to the trace distance as an objective function, we nonetheless see an overall decay in the gradients as we increase the number of hidden units of the model. We also see a similar decay occurring when we consider the variance of the $\infty$ norm of the gradient vector (see Appendix 5).

\section{CONCLUSION}

We show that for Haar random pure states and thermal states of random Hamiltonians, the gradient of an observable objective function vanishes exponentially with the number of hidden units. This shows that common types of QNNs are not only generically difficult to train via local optimization methods but also that the addition of hidden units will not always increase the power of QNNs. Indeed, asymptotically we see that unless the states generated satisfy an area law, such hidden neurons will likely be harmful.

One can prevent these entanglement-induced barren plateaus by violating any of the assumptions in our proofs. The first is to choose an atypical initial state: this has already been explored in Ref. [39]. Next, one could try to depart from the use of gradient-based optimization to train such quantum models. However, it is unlikely that, without knowledge of the global properties of the training objective function, such methods would succeed in light of Proposition 2. Lastly, one can train models using an objective function that does not correspond to an observable and is independent of the density operator.

Of the three approaches, it is this last approach that we advocate greater attention should be paid to in quantum machine learning. One tactic that can be used to circumvent our pessimistic results is to begin a discriminative learning task by first training generatively according to a quantity such as the quantum relative entropy $[8,19]$, which is nonlinear in the quantum state $\rho$. We will show in subsequent work that this quantum generative pretraining approach can be used to successfully train both Boltzmann machines and unitary networks and thereby mitigate some of the challenges identified here for training deep QNNs.

As a final point, it is important to recognize that while entanglement is a powerful tool to add to our models, it 
(a)

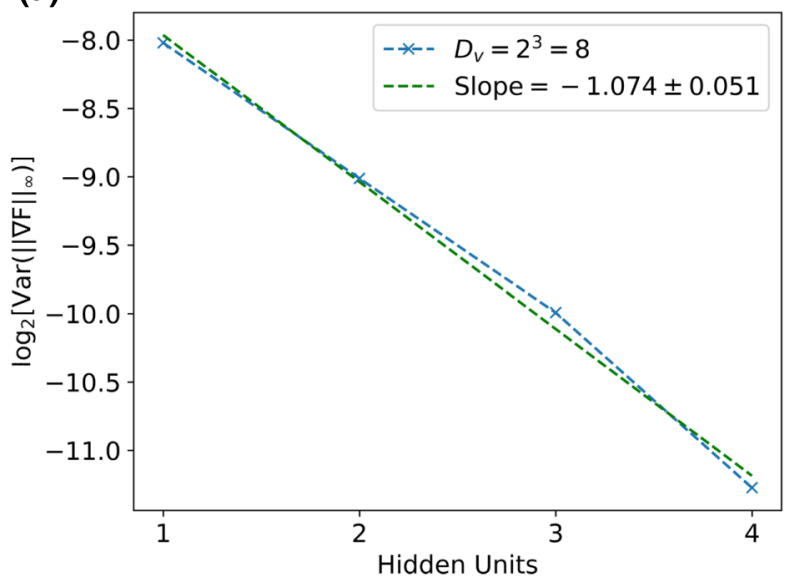

(b)

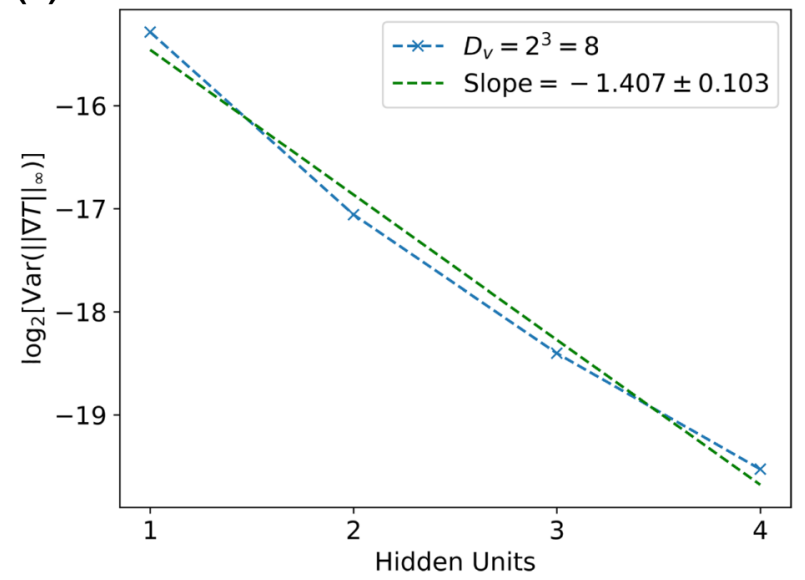

FIG. 5. (a),(b) A semilog plot highlighting the decay in the variance of the $\infty$ norm of the gradient vector over an ensemble of initialized models. The dashed blue line represents the variance over 1000 model instances. The dashed green line represents is the best fit obtained from least squares with the standard error of the estimated slope under the assumption of residual normality: (a) gradient estimates for the unitary model; (b) gradient estimates for the normalized quantum Boltzmann machine.

must be used like a scalpel and not a sledgehammer. Quantum properties such as entanglement may be harmful if not surgically deployed and judicially used. Careful integration of quantum effects into models will be necessary if we wish to realize the promised gains of quantum machinelearning algorithms [40].

\section{ACKNOWLEDGMENTS}

We thank Michael Bremner, Jarrod McClean, and Alessandro Rogero for helpful discussions and feedback. M.K. acknowledges funding from Australian Research Council (ARC) Centre of Excellence for Quantum Computation and Communication Technology (CQC2T), (Grant No. CE170100012). Support for C.O.M. and N.W. for the numerical studies was provided by the Laboratory Directed Research and Development Program at Pacific Northwest National Laboratory, a multiprogram national laboratory operated by Battelle for the U.S. Department of Energy, Release No. PNNL-SA-157287, and the theoretical work on this project by N.W. was supported by the U.S. Department of Energy, Office of Science, National Quantum Information Science Research Centers, Co-Design Center for Quantum Advantage under Grant No. DE-SC0012704. Additional support for N.W. was provided by a Google Research Award.

\section{APPENDIX A: PROOF OF UNITARY NETWORK GRADIENT}

Here, we provide a complete proof of Theorem 3 .

Theorem 6 (Gradient in Unitary Networks.). Assume that $\rho(\theta)$ is drawn from a unitary 2-design, where $\rho(\theta)$ is generated through a unitary ansatz of the form

$$
\rho(\theta)=\prod_{j=1}^{N} e^{-i H_{j} \theta_{j}}|0\rangle\langle 0| \prod_{j=N}^{1} e^{i H_{j} \theta_{j}}
$$

that acts on a Hilbert space that is the product of a hidden and visible space of dimensions $D_{h}$ and $D_{v}$, respectively. Further, define that $H_{k}(\theta)=\prod_{j=1}^{k} e^{-i H_{j} \theta_{j}} H_{k} \prod_{j=k}^{1} e^{i H_{j} \theta_{j}}$ for each $k$ obeys $\mathbb{E}\left[H_{k}(\theta) \rho(\theta)\right]=\mathbb{E}\left[H_{k}(\theta)\right] \mathbb{E}[\rho(\theta)]$. We then have that

$$
\mathbb{E}\left(\left|\operatorname{Tr}\left\{O_{o b j} \operatorname{Tr}_{h}[\rho(\theta)]\right\}\right|\right)
$$

is a Lipshitz continuous function of $\theta$ with constant $\Lambda$, obeying

$$
\Lambda \in O\left(\left\|O_{o b j}\right\|_{\infty}\left\|H_{k}\right\|_{\infty} \sqrt{\frac{D_{v}}{D_{h}}}\right) .
$$

Proof. In the above theorem, we introduce the quality $H_{k}(\theta)$ that concatenates $H_{k}$ with a part of the circuit. This quality arises when computing gradients of the objective function. To prove the above theorem, we first use the definition that

$$
\begin{aligned}
\rho\left(\theta+\delta_{k}\right):= & \left(\prod_{j=1}^{k} e^{-i H_{j} \theta_{j}} e^{-i H_{k} \delta_{k}} \prod_{j>k} e^{-i H_{j} \theta_{j}}\right) \rho_{0} \\
& \times\left(\prod_{j=1}^{k} e^{-i H_{j} \theta_{j}} e^{-i H_{k} \delta_{k}} \prod_{j>k} e^{-i H_{j} \theta_{j}}\right)^{\dagger} .
\end{aligned}
$$


We then wish to analyze the distribution over $\theta$ of $\left|\operatorname{Tr}_{v}\left\{O_{\mathrm{obj}} \operatorname{Tr}_{h}\left[\rho(\theta)-\rho\left(\theta+\delta_{k}\right)\right]\right\}\right|$, under the assumption that the unitaries satisfy a 2-design condition.

Using Hadamard's lemma, we can express the difference between the expectation values as

$$
\begin{gathered}
\operatorname{Tr}_{v}\left\{O_{\mathrm{obj}} \operatorname{Tr}_{h}\left[\rho(\theta)-\left(\prod_{j=1}^{k} e^{-i H_{j} \theta_{j}} e^{-i H_{k}\left|\delta_{k}\right|} \prod_{j=k}^{1} e^{i H_{j} \theta_{j}}\right) \rho(\theta)\left(\prod_{j=1}^{k} e^{-i H_{j} \theta_{j}} e^{-i H_{k}\left|\delta_{k}\right|} \prod_{j=k}^{1} e^{i H_{j} \theta_{j}}\right)^{\dagger}\right]\right\} \\
:=\left\{\operatorname{Tr}_{v}\left[O_{\mathrm{obj}} \operatorname{Tr}_{h}\left(\rho(\theta)-e^{-i H_{k}(\theta)\left|\delta_{k}\right|} \rho(\theta) e^{i H_{k}(\theta)\left|\delta_{k}\right|}\right)\right]\right\}=\operatorname{Tr}_{v}\left(O_{\mathrm{obj}} \sum_{q=1}^{\infty} \frac{\operatorname{Tr}_{h}\left\{\operatorname{Ad}_{-i H_{k}(\theta)}^{q}[\rho(\theta)]\right\}\left|\delta_{k}\right|^{q}}{q !}\right) .
\end{gathered}
$$

Next, making the assumption that $H_{k}(\theta)$ and $\rho(\theta)$ are uncorrelated, we can further simplify this result. Our exposition now follows that of Popescu et al. [20]; which we modify to show that a concentration of measure exists for the commutators of $H_{k}(\theta)$ and $\rho(\theta)$.

We now work under the assumption that the expectation values are independent. We further denote the expectation value over the Hamiltonian as $\mathbb{E}_{H}$ and the expectation value over the state as $\mathbb{E}_{\phi}$. If this independence assumption holds, then we need to argue about the magnitude of terms of the form $\mathbb{E}_{\phi}\left(\operatorname{Tr}_{v}\left\{\operatorname{Tr}_{h}\left[H_{k}(\theta) \rho(\theta)\right]^{2}\right\}\right)$. We can estimate this by introducing two copies of the quantum state and linking both terms through the use of a flip operator $F_{v v^{\prime}}$ such that

$$
F_{v v^{\prime}}=\sum_{v v^{\prime}}\left|v^{\prime}\right\rangle\langle v|\otimes| v\rangle\left\langle v^{\prime}\right|
$$

In the following, we use this notation of primed indices to refer to the visible and hidden subsystems of the first and second copies, respectively.

The commutators in general consist of many different products of $H_{k}$ and the state operator. Below, we argue about their form in generality. Let us assume that $p_{1}, p_{2}, q_{1}, q_{2}$ are positive integers. We then wish to compute the product of traces of the form $\operatorname{Tr}_{h}\left(H_{k}(\theta)^{p_{1}} \rho H_{k}(\theta)^{p_{2}}\right) \operatorname{Tr}_{h}\left[H_{k}(\theta)^{q_{1}} \rho H_{k}(\theta)^{q_{2}}\right]$. By applying the flip operator and taking the quantum state $\rho(\theta)$ to be $|\phi\rangle\langle\phi|$,

$$
\begin{aligned}
\operatorname{Tr} & \left.\mathbb{E}_{\phi}\left\{\operatorname{Tr}_{h}\left[H_{k}(\theta)^{p_{1}}\right] \rho H_{k}(\theta)^{p_{2}} \operatorname{Tr}_{h}\left[H_{k}(\theta)^{q_{1}} \rho H_{k}(\theta)^{q_{2}}\right]\right\}\right) \\
& =\operatorname{Tr}_{v v^{\prime}}\left[\mathbb{E}_{\phi}\left(\left\{\operatorname{Tr}_{h}\left[H_{k}^{p_{1}}(\theta) \rho(\theta) H_{k}^{p_{2}}(\theta)\right] \otimes \operatorname{Tr}_{h}\left[H_{k}^{q_{1}}(\theta) \rho(\theta) H_{k}^{q_{2}}(\theta)\right]\right\} F_{v v^{\prime}}\right)\right] \\
& =\operatorname{Tr}_{v v^{\prime}} \operatorname{Tr}_{h h^{\prime}}\left[\mathbb{E}_{\phi}\left(\left\{\operatorname{Tr}_{h}\left[H_{k}^{p_{1}}(\theta) \rho(\theta) H_{k}^{p_{2}}(\theta)\right] \otimes \operatorname{Tr}_{h}\left[H_{k}^{q_{1}}(\theta) \rho(\theta) H_{k}^{q_{2}}(\theta)\right]\right\}\left(F_{v v^{\prime}} \otimes I\right)\right)\right] \\
& =\operatorname{Tr}\left(\mathbb{E}_{\phi}\left\{(|\phi\rangle\langle\phi|\otimes| \phi\rangle\langle\phi|)\left[H_{k}^{p_{1}}(\theta) \otimes H_{k}^{q_{1}}(\theta)\right]\left(F_{v v^{\prime}} \otimes I\right)\left[H_{k}^{p_{2}}(\theta) \otimes H_{k}^{q_{2}}(\theta)\right]\right\}\right) .
\end{aligned}
$$

The next step in this is to recognize that if $|\phi\rangle\langle\phi|$, the above tensor products are a symmetric quantum state. Therefore, if we express the state as the sum of its antisymmetric component and its symmetric component, then the antisymmetric component must be zero [20]. We then see from the fact that $\rho(\theta)$ is assumed to be drawn from a unitary 2-design that the expectation value is unitarily invariant and we can then follow the arguments laid out in Ref. [20], that

$$
\begin{aligned}
\operatorname{Tr} & \left.\mathbb{E}_{\phi}\left\{\operatorname{Tr}_{h}\left[H_{k}(\theta)^{p_{1}}\right] \rho H_{k}(\theta)^{p_{2}} \operatorname{Tr}_{h}\left[H_{k}(\theta)^{q_{1}} \rho H_{k}(\theta)^{q_{2}}\right]\right\}\right) \\
& =\frac{2 D^{2}}{D(D+1)} \operatorname{Tr}\left\{\left[\left(\frac{\Pi^{\mathrm{sym}}}{D^{2}}\right)\left[H_{k}^{p_{1}}(\theta) \otimes H_{k}^{q_{1}}(\theta)\right]\right.\right. \\
& \left.\left.\times\left(F_{v v^{\prime}} \otimes I\right)\left[H_{k}^{p_{2}}(\theta) \otimes H_{k}^{q_{2}}(\theta)\right]\right]\right\} .
\end{aligned}
$$


Next, if we define the flip operator on the dilated space including the hidden and visible units to be $F_{r r^{\prime}}=F_{v v^{\prime}} \otimes F_{h h^{\prime}}$, we can then express $\Pi^{\mathrm{sym}}=\frac{1}{2}\left(I+F_{r r^{\prime}}\right)$. Finally, using the properties of the flip operator, we find that we can write

$$
\begin{aligned}
\operatorname{Tr}_{v}\left(\mathbb{E}_{\phi}\left\{\operatorname{Tr}_{h}\left[H_{k}(\theta)^{p_{1}}\right] \rho H_{k}(\theta)^{p_{2}} \operatorname{Tr}_{h}\left[H_{k}(\theta)^{q_{1}} \rho H_{k}(\theta)^{q_{2}}\right]\right\}\right) \\
=\frac{D^{2}}{D(D+1)} \operatorname{Tr}\left(\frac{\left[H_{k}^{p_{1}}(\theta) \otimes H_{k}^{q_{1}}(\theta)\right]\left(F_{v v^{\prime}} \otimes I\right)\left[H_{k}^{p_{2}}(\theta) \otimes H_{k}^{q_{2}}(\theta)\right]}{D^{2}}\right) \\
+\frac{D^{2}}{D(D+1)} \operatorname{Tr}\left(\frac{\left(F_{v v^{\prime}} \otimes F_{h h^{\prime}}\right)\left[H_{k}^{p_{1}}(\theta) \otimes H_{k}^{q_{1}}(\theta)\right]\left(F_{v v^{\prime}} \otimes I\right)\left[H_{k}^{p_{2}}(\theta) \otimes H_{k}^{q_{2}}(\theta)\right]}{D^{2}}\right) \\
=\frac{D^{2}}{D(D+1)} \operatorname{Tr}\left(\frac{\left[H_{k}^{p_{1}}(\theta) \otimes H_{k}^{q_{1}}(\theta)\right]\left(F_{v v^{\prime}} \otimes I\right)\left[H_{k}^{p_{2}}(\theta) \otimes H_{k}^{q_{2}}(\theta)\right]}{D^{2}}\right) \\
+\frac{D^{2}}{D(D+1)} \operatorname{Tr}\left(\frac{\left[H_{k}^{q_{1}}(\theta) \otimes H_{k}^{p_{1}}(\theta)\right]\left(I \otimes F_{h h^{\prime}}\right)\left[H_{k}^{p_{2}}(\theta) \otimes H_{k}^{q_{2}}(\theta)\right]}{D^{2}}\right) \\
=\frac{D^{2}}{D(D+1)} \operatorname{Tr}\left(\frac{\left(F_{v v^{\prime}} \otimes I\right)\left[H_{k}^{p_{1}+p_{2}}(\theta) \otimes H_{k}^{q_{1}+q_{2}}(\theta)\right]}{D^{2}}\right)+\frac{D^{2}}{D(D+1)} \operatorname{Tr}^{2}\left(\frac{\left(I \otimes F_{h h^{\prime}}\right)\left[H_{k}^{q_{1}+p_{2}}(\theta) \otimes H_{k}^{p_{1}+q_{2}}(\theta)\right]}{D^{2}}\right) \\
=\frac{D^{2}}{D(D+1)} \operatorname{Tr}_{v}\left(\frac{\operatorname{Tr}_{h}\left[H_{k}^{p_{1}+p_{2}}(\theta)\right] \operatorname{Tr}_{h}\left[H_{k}^{q_{1}+q_{2}}(\theta)\right]}{D^{2}}\right)+\frac{D^{2}}{D(D+1)} \operatorname{Tr}_{h}\left(\frac{\operatorname{Tr}_{v}\left[H_{k}^{q_{1}+p_{2}}(\theta)\right] \operatorname{Tr}_{v}\left[H_{k}^{p_{1}+q_{2}}(\theta)\right]}{D^{2}}\right)
\end{aligned}
$$

Therefore, we have from the triangle inequality that

$$
\begin{aligned}
& \left|\operatorname{Tr}_{v}\left(\mathbb{E}_{\phi}\left\{\operatorname{Tr}_{h}\left[H_{k}(\theta)^{p_{1}}\right] \rho H_{k}(\theta)^{p_{2}} \operatorname{Tr}_{h}\left[H_{k}(\theta)^{q_{1}} \rho H_{k}(\theta)^{q_{2}}\right]\right\}\right)-\frac{D^{2}}{D(D+1)} \operatorname{Tr}_{v}\left(\frac{\operatorname{Tr}_{h}\left[H_{k}^{p_{1}+p_{2}}(\theta)\right] \operatorname{Tr}_{h}\left[H_{k}^{q_{1}+q_{2}}(\theta)\right]}{D^{2}}\right)\right| \\
& \quad \leq\left|\operatorname{Tr}_{h}\left(\frac{\operatorname{Tr}_{v}\left[H_{k}^{q_{1}+p_{2}}(\theta)\right] \operatorname{Tr}_{v}\left[H_{k}^{p_{1}+q_{2}}(\theta)\right]}{D^{2}}\right)\right| \leq \frac{\operatorname{Tr}\left[\left|H_{k}^{q_{1}+q_{2}}(\theta)\right|\right]\left\|\operatorname{Tr}_{v}\left[H_{k}(\theta)^{q_{1}+q_{2}}\right]\right\|_{\infty}}{D^{2}} \leq \frac{\left\|H_{k}\right\|_{\infty}^{p_{1}+p_{2}+q_{1}+q_{2}}}{D_{h}} .
\end{aligned}
$$

Here, the last inequality follows from the fact that the Schatten $\infty$ norm is unitarily invariant and thus $\left\|H_{k}(\theta)\right\|_{\infty}=$ $\left\|H_{k}\right\|_{\infty}$.

Next, let us consider the expectation value for one of the terms in the expansion

$$
\begin{aligned}
\mathbb{E} & \left(\left\|\operatorname{Tr}_{h}\left\{\operatorname{Ad}_{-i H_{k}(\theta)}^{q}[\rho(\theta)]\right\}\right\|_{1}\right) \\
& \leq \mathbb{E}\left(\sqrt{D_{v}}\left\|\operatorname{Tr}_{h}\left\{\operatorname{Ad}_{-i H_{k}(\theta)}^{q}[\rho(\theta)]\right\}\right\|_{2}\right) \\
& \leq \sqrt{D_{v} \mathbb{E}\left(\left\|\operatorname{Tr}_{h}\left\{\operatorname{Ad}_{H_{k}(\theta)}^{q}[\rho(\theta)]\right\}\right\|_{2}^{2}\right)} .
\end{aligned}
$$

Every term in $\operatorname{Ad}_{H_{k}(\theta)}^{q}[\rho(\theta)]$ consists of $q H_{k}(\theta)$ and, further, the $2^{q-1}$ terms have a positive coefficient and the $2^{q-1}$ terms have a negative coefficient. The proof of this fact is inductive. For $q=1$,

$$
\operatorname{Ad}_{H_{k}(\theta)}[\rho(\theta)]=H_{k}(\theta) \rho(\theta)-\rho(\theta) H_{k}(\theta),
$$

which demonstrates the base case of $q=1$. Now assume that the claim is valid for $q=p$. We then have that

$$
\operatorname{Ad}_{H_{k}(\theta)}^{p+1}[\rho(\theta)]=H_{k}(\theta) \operatorname{Ad}_{H_{k}(\theta)}^{p}[\rho(\theta)]-\operatorname{Ad}_{H_{k}(\theta)}^{p}[\rho(\theta)] H_{k}(\theta)
$$

The induction step follows immediately from this observation and it is clear that the claim is valid for all $q$.

Now, if we expand $\left(\operatorname{Tr}_{h}\left\{\operatorname{Ad}_{H_{k}(\theta)}^{q}[\rho(\theta)]\right\}\right)^{2}$ using the linearity of the partial-trace operation, we find that each term is of the form $\operatorname{Tr}_{h}\left[H_{k}^{p_{1}}(\theta) \rho(\theta) H_{k}^{p_{2}}(\theta)\right] \operatorname{Tr}_{h}\left[H_{k}^{q_{1}}(\theta) \rho(\theta) H_{k}^{q_{2}}(\theta)\right]$, where $p_{1}+p_{2}=q=q_{1}+q_{2}$. The expression in Eq. (A7) then shows us that we can replace each term with $\left[D^{2} / D(D+1)\right] \operatorname{Tr}_{v}\left(\left\{\operatorname{Tr}_{h}\left[H_{k}^{q}(\theta)\right]\right\}^{2} / D^{2}\right)$ while incurring a small error. Importantly, this value is independent of $p_{1}, p_{2}, q_{1}, q_{2}$. Thus, since there are $2^{q-1}$ such terms with a negative coefficient 
and $2^{q-1}$ with a positive coefficient for each of the partial traces, there are similarly $2^{2 q-1}$ terms with a negative coefficient in the expansion and $2^{2 q-1}$ with a positive coefficient. Ergo, the sums over all such terms present in the adjoint are zero up to the small error terms given in Eq. (A7). Thus we have that

$$
\sqrt{D_{v} \mathbb{E}\left(\| \operatorname{Tr}_{h}\left\{\operatorname{Ad}_{H_{k}(\theta)}^{q}[\rho(\theta)] \|_{2}^{2}\right\}\right)} \leq\left(2\left\|H_{k}\right\|_{\infty}\right)^{q} \sqrt{\frac{D_{v}}{D_{h}}} .
$$

Next, from Eq. (A10), we have that

$$
\begin{aligned}
\mathbb{E} \mid & \operatorname{Tr}_{v}\left(O_{\text {obj }} \sum_{q=1}^{\infty} \frac{\operatorname{Tr}_{h}\left\{\operatorname{Ad}_{-i H_{k}(\theta)}^{q}[\rho(\theta)]\right\}\left|\delta_{k}\right|^{q}}{q !}\right) \mid \\
& \leq\left\|O_{\text {Obj }}\right\|_{\infty} \sum_{q=1}^{\infty} \mathbb{E}\left(\left\|\frac{\operatorname{Tr}_{h}\left\{\operatorname{Ad}_{-i H_{k}(\theta)}^{q}[\rho(\theta)]\right\}\left|\delta_{k}\right|^{q}}{q !}\right\|_{1}\right) \\
& \leq\left\|O_{\text {Obj }}\right\|_{\infty} \sum_{q=1}^{\infty} \sqrt{D_{v} \mathbb{E}\left(\left\|\frac{\operatorname{Tr}_{h}\left\{\operatorname{Ad}_{-i H_{k}(\theta)}^{q}[\rho(\theta)]\right\}\left|\delta_{k}\right|^{q}}{q !}\right\|_{2}^{2}\right)} \\
& \leq\left|\delta_{k}\right|\left\|O_{\text {obj }}\right\|_{\infty}\left\|H_{k}\right\|_{\infty} e^{2\left\|H_{k}\right\| \|_{\infty}\left|\delta_{k}\right|} \sqrt{\frac{D_{v}}{D_{h}}}, \\
& \in O\left(\left|\delta_{k}\right|\left\|O_{\mathrm{Obj}}\right\|_{\infty}\left\|H_{k}\right\|_{\infty} \sqrt{\frac{D_{v}}{D_{h}}}\right)
\end{aligned}
$$

where we use the assumption that $\left\|H_{k}\right\|_{\infty}\left|\delta_{k}\right| \in O(1)$. From this, our claim about the Lipshitz constant follows immediately from the definition of Lipshitz continuity and from Eq. (A2).

\section{APPENDIX B: PROOF OF QUANTUM BOLTZMANN MACHINE GRADIENT}

Here, we provide a complete proof for the gradient of a quantum Boltzmann machine. We can always assume that the Hamiltonian is traceless. Indeed, for any Hamiltonian $H^{\prime}$ with a nonzero trace, we can introduce a Hamiltonian $H=H^{\prime}-\alpha \nVdash$ such that $\operatorname{Tr}(H)=0$ and $H$ leads to the same thermal state:

$$
\rho_{\text {thermal }}=\frac{e^{-H t}}{\operatorname{Tr}\left(e^{-H t}\right)}=\frac{e^{-H^{\prime} t+\alpha \nVdash t}}{\operatorname{Tr}\left(e^{-H^{\prime} t+\alpha \nVdash t}\right)}=\frac{e^{\alpha t} e^{-H^{\prime} t}}{e^{\alpha t} \operatorname{Tr}\left(e^{-H^{\prime} t}\right)}=\frac{e^{-H^{\prime} t}}{\operatorname{Tr}\left(e^{-H^{\prime} t}\right)} .
$$

Theorem 7 (Gradient for Boltzmann Machines.). Assume that $H \in \mathbb{C}^{D \times D}$ is a random Hermitian matrix drawn from an ensemble in the following manner: a diagonal matrix with eigenvalues $E_{j} \in \mathbb{R}$ chosen according to a probability $\operatorname{Pr}\left(E_{j}\right)$ such that $\max _{k} \mathbb{E}\left(1 / D^{2}\left(\sum_{j \neq k}\left(E_{j}-E_{k}\right)^{-1}\right)^{2}\right) \leq \Gamma^{2}$ and is then conjugated with a unitary drawn from a distribution that is a unitary 2-design. Let $H_{k}=h_{v} \otimes h_{h} \in \mathbb{C}^{D \times D}$ where $h_{v}, h_{h}$ are fixed Hermitians acting on the visible and hidden subspace, respectively, and

$$
\rho\left(\theta_{k}\right):=e^{-\left(H+\theta_{k} H_{k}\right)} / \operatorname{Tr}\left(e^{-\left(H+\theta_{k} H_{k}\right)}\right) .
$$

Finally, let $O_{o b j} \in \mathbb{C}^{D_{v} \times D_{v}}$ be a Hermitian matrix. Then

$$
\kappa:=\operatorname{Tr}\left\{\left(O_{o b j} \otimes I_{h}\right)\left[\rho\left(\theta_{k}\right)\right]\right\}
$$

is a differentiable function that obeys

$$
\left|\frac{\partial \kappa}{\partial \theta_{k}}\right|_{\theta_{k}=0} \in O\left(\left\|O_{o b j}\right\|_{\infty} \Gamma\left\|H_{k}\right\|_{\infty} \sqrt{\left.\frac{D_{v}}{D_{h}}\left(\frac{\operatorname{Tr}\left(h_{h}\right)^{2}}{D_{v} \operatorname{Tr}\left(h_{h}^{2}\right)}+1\right)\right)},\right.
$$

with high probability over the ensemble. 
Proof. First, note that if we begin with an observable $O_{\text {obj }}$ acting on the visible subspace, then the difference between the observable for $\rho(\theta)=e^{-\left(H+\theta_{k} H_{k}\right)} / Z\left(\theta_{k}\right)$ and $\rho(\theta):=\rho$ reads as follows

$$
\begin{aligned}
\mathbb{E}\left\{\left|\operatorname{Tr}\left[\left(O_{\text {obj }} \otimes I_{h}\right) \rho\left(\theta_{k}\right)\right]-\operatorname{Tr}\left[\left(O_{\text {obj }} \otimes I_{h}\right) \rho\right]\right|\right\} & \leq\left\|O_{\text {Obj }}\right\|_{\infty} \mathbb{E}\left\{\left\|\operatorname{Tr}_{h}\left[\rho\left(\theta_{k}\right)-\rho\right]\right\|_{1}\right\} \\
& \leq\left\|O_{\text {Obj }}\right\|_{\infty} \sqrt{D_{v} \mathbb{E}\left\{\left\|\operatorname{Tr}_{h}\left[\rho\left(\theta_{k}\right)-\rho\right]\right\|_{2}^{2}\right\}}
\end{aligned}
$$

Therefore, just like the unitary network case, we now focus our attention on bounding the expectation value of the difference between the density operators. The main difference here is that the density operators are defined via imaginary time evolution rather than real time.

From Taylor's theorem, we have that if the Hamiltonian $H+s H_{k}$ has no level crossings on the interval $s \in\left[0, \theta_{k}\right]$ then, to order $O\left(\theta_{k}^{2}\right)$, the eigenvectors of $H+\theta_{k} H_{k}$ can be identified using perturbation theory. In particular, for any $p \in$ $\{0, \ldots, D-1\}$, let $|p\rangle$ be an eigenvector of $H$ with eigenvalue $E_{p}$. Then, the eigenvector $\left|n^{\prime}\right\rangle$ of $H+\theta_{k}$ that corresponds to the eigenvector $|n\rangle$ of $H$ can be expressed as

$$
\left|n^{\prime}\right\rangle=|n\rangle+\theta_{k} \sum_{j \neq n} \frac{|j\rangle\left\langle j\left|H_{k}\right| n\right\rangle}{E_{n}-E_{j}}+O\left(\theta_{k}^{2}\right)
$$

This implies that

$$
\operatorname{Tr}_{h}\left(\left|n^{\prime}\right\rangle\left\langle n^{\prime}|-| n\right\rangle\langle n|\right)=\operatorname{Tr}_{h}\left(\theta_{k} \sum_{j \neq n} \frac{|j\rangle\left\langle j\left|H_{k}\right| n\right\rangle\langle n|}{E_{n}-E_{j}}+\frac{|n\rangle\left\langle n\left|H_{k}\right| j\right\rangle\langle j|}{E_{n}-E_{j}}\right)+O\left(\theta_{k}^{2}\right)
$$

Next, let us write, for any $\ell \in\{0, \ldots, D-1\}$, the eigenvector $|\ell\rangle=\sum_{p q} \alpha_{p q}^{(\ell)}|p q\rangle$, where $\alpha_{p q}^{(\ell)}$ is a complex number and $|p q\rangle:=|p\rangle_{v} \otimes|q\rangle_{h}$ for some appropriate basis for the visible and hidden subsystems. The expectation value over the state vectors can then be thought of as an average of these coefficients.

We use the fact that $H_{k}=h_{v} \otimes H_{h}$ to choose the bases of the visible and hidden subsystems to diagonalize $h_{v}$ and $h_{h}$. Thus we can state $h_{v} \otimes h_{h}|p q\rangle:=\lambda_{p q}|p q\rangle$ for $\lambda_{p q} \in \mathbb{R}$.

With these choices in place, we can write

$$
\begin{gathered}
\operatorname{Tr}_{h}\left(\sum_{j \neq n} \frac{|j\rangle\left\langle j\left|H_{k}\right| n\right\rangle\langle n|}{E_{n}-E_{j}}\right) \\
=\sum_{j \neq n} \operatorname{Tr}_{h}\left(\sum_{p q r s t u v w} \frac{\alpha_{p q}^{(j)} \alpha_{r s}^{*(j)} \alpha_{t u}^{(n)} \alpha_{v w}^{*(n)}}{E_{n}-E_{j}}|p q\rangle\left\langle r s\left|H_{k}\right| t u\right\rangle\langle v w|\right) \\
=\sum_{j \neq n} \operatorname{Tr}_{h}\left(\sum_{p q r s v w} \frac{\alpha_{p q}^{(j)} \alpha_{r s}^{*(j)} \alpha_{r s}^{(n)} \alpha_{v w}^{*(n)} \lambda_{r s}}{E_{n}-E_{j}}|p q\rangle\langle v w|\right) \\
=\sum_{j \neq n}\left(\sum_{p q r s v} \frac{\alpha_{p q}^{(j)} \alpha_{r s}^{*(j)} \alpha_{r s}^{(n)} \alpha_{v q}^{*(n)} \lambda_{r s}}{E_{n}-E_{j}}|p\rangle\langle v|\right) \\
\operatorname{Tr}_{h}\left(\left|n^{\prime}\right\rangle\left\langle n^{\prime}|-| n\right\rangle\langle n|\right)^{2} \\
=\left[\sum_{j \neq n}\left(\sum_{p q r s v} \frac{\left(\alpha_{p q}^{(j)} \alpha_{r s}^{*(j)} \alpha_{r s}^{(n)} \alpha_{v q}^{*(n)}+\alpha_{p q}^{(n)} \alpha_{r s}^{*(n)} \alpha_{r s}^{(j)} \alpha_{v q}^{*(j)}\right) \lambda_{r s}}{E_{n}-E_{j}}|p\rangle\langle v|\right)\right]^{2}+O\left(\theta_{k}^{4}\right)
\end{gathered}
$$




$$
\begin{aligned}
& =\sum_{j, j^{\prime} \neq n} \sum_{\substack{p q r s v \\
q^{\prime} r^{\prime} s^{\prime} v^{\prime}}} \frac{\left(\alpha_{p q}^{(j)} \alpha_{r s}^{*(j)} \alpha_{r s}^{(n)} \alpha_{v q}^{*(n)}+\alpha_{p q}^{(n)} \alpha_{r s}^{*(n)} \alpha_{r s}^{(j)} \alpha_{v q}^{*(j)}\right)\left(\alpha_{v q^{\prime}}^{(j)} \alpha_{r^{\prime} s^{\prime}}^{*(j)} \alpha_{r^{\prime} s^{\prime}}^{(n)} \alpha_{v^{\prime} q^{\prime}}^{*(n)}+\alpha_{v q^{\prime}}^{(n)} \alpha_{r^{\prime} s^{\prime}}^{*(n)} \alpha_{r^{\prime} s^{\prime}}^{(j)} \alpha_{v^{\prime} q^{\prime}}^{*(j)}\right) \lambda_{r s} \lambda_{r^{\prime} s^{\prime}}}{\left(E_{n}-E_{j}\right)\left(E_{n}-E_{j^{\prime}}\right)}|p\rangle\left\langle v^{\prime}\right| \\
& +O\left(\theta_{k}^{4}\right) \text {. }
\end{aligned}
$$

There is a total of eight terms that arise when we expand the above products. Let us consider the first case that emerges in the expectation value of the trace of the previous result. Here, we invoke the fact that the eigenvectors are sampled from a unitary 2-design, which means that any quantity that is at most quadratic in the probability will have an expectation value that coincides with the Haar average. A final point to note is that as a consequence of unitary invariance and the discussion contained in Ref. [38, Appendix A], the expectation value of the product of any two terms is zero unless all of their indices match. Further, up to relative errors that are $O(1 / D)$, the expectation values of the coefficients are independent of each other. This implies that if $\operatorname{Tr}\left(h_{h}\right)=0$, then

$$
\begin{aligned}
& \mathbb{E}\left(\sum_{j, j^{\prime} \neq n} \sum_{\substack{p q r v v \\
q^{\prime} r^{\prime} s^{\prime} v^{\prime}}} \frac{\alpha_{p q}^{(j)} \alpha_{r s}^{*(j)} \alpha_{r s}^{(n)} \alpha_{v q}^{*(n)} \alpha_{v q^{\prime}}^{(j)} \alpha_{r^{\prime} s^{\prime}}^{*(j)} \alpha_{r^{\prime} s^{\prime}}^{(n)} \alpha_{p q^{\prime}}^{*(n)} \lambda_{r s} \lambda_{r^{\prime} s^{\prime}}}{\left(E_{n}-E_{j}\right)\left(E_{n}-E_{j^{\prime}}\right)}\right) \\
& =O\left[\left(\sum_{j, j^{\prime} \neq n} \sum_{p q q^{\prime}} \frac{\mathbb{E}\left(\left|\alpha_{p q}^{(j)}\right|^{2}\right) \mathbb{E}\left(\left|\alpha_{p q}^{(n)}\right|^{2}\right) \mathbb{E}\left(\left|\alpha_{p q^{\prime}}^{\left(j^{\prime}\right)}\right|^{2}\right) \mathbb{E}\left(\left|\alpha_{p q^{\prime}}^{(n)}\right|^{2}\right) \lambda_{p q} \lambda_{p q^{\prime}}}{\left(E_{n}-E_{j}\right)\left(E_{n}-E_{j^{\prime}}\right)}\right)\right. \\
& +\left(\sum_{j, j^{\prime} \neq n} \sum_{p q v} \frac{\mathbb{E}\left(\left|\alpha_{p q}^{(j)}\right|^{2}\right) \mathbb{E}\left(\left|\alpha_{p q}^{(n)}\right|^{2}\right) \mathbb{E}\left(\left|\alpha_{v q}^{(n)}\right|^{2}\right) \mathbb{E}\left(\left|\alpha_{p q}^{\left(j^{\prime}\right)}\right|^{2}\right) \lambda_{p q}^{2}}{\left(E_{n}-E_{j}\right)\left(E_{n}-E_{j^{\prime}}\right)}\right) \\
& \left.+\left(\sum_{j, j^{\prime} \neq n} \sum_{p q} \frac{\mathbb{E}\left(\left|\alpha_{p q}^{(n)}\right|^{4}\right) \mathbb{E}\left(\left|\alpha_{p q}^{(j)}\right|^{2}\right) \mathbb{E}\left(\left|\alpha_{p q}^{\left(j^{\prime}\right)}\right|^{2}\right) \lambda_{p q}^{2}}{\left(E_{n}-E_{j}\right)\left(E_{n}-E_{j^{\prime}}\right)}\right)\right] \\
& =O\left[\frac{1}{D^{4}}\left(\sum_{j \neq n} \frac{1}{E_{n}-E_{j}}\right)^{2}\left(\left(\operatorname{Tr}\left(h_{v}^{2}\right)\left[\operatorname{Tr}\left(h_{h}\right)\right]^{2}+\left(D_{v}+1\right) \operatorname{Tr}\left(H_{k}^{2}\right)\right)\right]\right. \\
& =O\left[\frac{1}{D^{4}}\left(\sum_{j \neq n} \frac{1}{E_{n}-E_{j}}\right)^{2}\left(\frac{\operatorname{Tr}\left(h_{h}\right)^{2}}{\operatorname{Tr}\left(h_{h}^{2}\right)}+D_{v}\right) \operatorname{Tr}\left(H_{k}^{2}\right)\right] \\
& =O\left[\frac{D_{v}}{D^{3}}\left(\sum_{j \neq n} \frac{1}{E_{n}-E_{j}}\right)^{2}\left(\frac{\operatorname{Tr}\left(h_{h}\right)^{2}}{D_{v} \operatorname{Tr}\left(h_{h}^{2}\right)}+1\right)\left\|H_{k}\right\|_{\infty}^{2}\right] .
\end{aligned}
$$

Now, under the assumption that

$$
\mathbb{E}\left[\left(\sum_{j \neq n} \frac{1}{E_{n}-E_{j}}\right)^{2}\right] \leq \Gamma^{2} D^{2},
$$

it follows that this term is asymptotically bounded above by $O\left(\Gamma^{2}\left\|H_{k}\right\|_{\infty}^{2} / D_{h}\right)$. It is straightforward to verify that the same bound holds for all remaining four products in the expansion.

It then follows from Eqs. (B2) and (B7) that

$$
\begin{aligned}
\left|\partial_{\theta_{k}}\left(\mathbb{E}\left\{\mid \operatorname{Tr}\left[\left(O_{\text {obj }} \otimes I_{h}\right) \rho\left(\theta_{k}\right)\right]\right\}\right)\right| & =\left|\lim _{\theta_{k} \rightarrow 0} \frac{\mathbb{E}\left(\left|\operatorname{Tr}\left[\left(O_{\text {obj }} \otimes I_{h}\right) \rho\left(\theta_{k}\right)\right]-\operatorname{Tr}\left[\left(O_{\text {obj }} \otimes I_{h}\right) \rho\right]\right|\right)}{\theta_{k}}\right| \\
& \in O\left(\left\|O_{\text {obj }}\right\|_{\infty} \Gamma\left\|H_{k}\right\|_{\infty} \sqrt{\frac{D_{v}}{D_{h}}\left(\frac{\operatorname{Tr}\left(h_{h}\right)^{2}}{D_{v} \operatorname{Tr}\left(h_{h}^{2}\right)}+1\right)}\right) .
\end{aligned}
$$


The claim that this bound on the derivative holds with high probability over the ensemble is then a direct consequence of Markov's inequality.

\section{APPENDIX C: NUMERICAL EXPERIMENTS: VARIANCE OF THE GRADIENT VECTOR}

In Fig. 5, we include some numerical experiments detailing the overall decay in variance of the $\infty$ norm of the gradient vector as we increase the number of hidden units of the unitary model and the quantum Boltzmann machine.

[1] S. Lloyd, M. Mohseni, and P. Rebentrost, Quantum principal component analysis, Nat. Phys. 10, 631 (2014).

[2] P. Rebentrost, M. Mohseni, and S. Lloyd, Quantum Support Vector Machine for Big Data Classification, Phys. Rev. Lett. 113, 130503 (2014).

[3] M. Schuld and N. Killoran, Quantum Machine Learning in Feature Hilbert Spaces, Phys. Rev. Lett. 122, 040504 (2019).

[4] V. Havlíček, A. D. Córcoles, K. Temme, A. W. Harrow, A. Kandala, J. M. Chow, and J. M. Gambetta, Supervised learning with quantum-enhanced feature spaces, Nature 567, 209 (2019).

[5] M. Schuld, I. Sinayskiy, and F. Petruccione, The quest for a quantum neural network, Quantum Inf. Process. 13, 2567 (2014).

[6] N. Wiebe, A. Kapoor, and K. M. Svore, Quantum deep learning, Quantum Inf. Comput. 16, 541 (2016).

[7] E. Farhi and H. Neven, Classification with quantum neural networks on near term processors. arXiv preprint ArXiv:1802.06002, 2018.

[8] N. Wiebe and L. Wossnig, Generative training of quantum Boltzmann machines with hidden units. arXiv preprint ArXiv:1905.09902, 2019.

[9] E. Tang, in Proceedings of the 51st Annual ACM SIGACT Symposium on Theory of Computing, 2019, p. 217.

[10] E. Tang, Quantum-inspired classical algorithms for principal component analysis and supervised clustering. arXiv preprint ArXiv:1811.00414, 2018.

[11] A.Gilyén, S. Lloyd, and E. Tang, Quantum-inspired lowrank stochastic regression with logarithmic dependence on the dimension. arXiv preprint ArXiv:1811.04909, 2018.

[12] J. R. McClean, S. Boixo, V. N. Smelyanskiy, R. Babbush, and $\mathrm{H}$. Neven, Barren plateaus in quantum neural network training landscapes, Nat. Commun. 9, 1 (2018).

[13] M. Cerezo, A. Sone, T. Volkoff, L. Cincio, and P. J. Coles, Cost-function-dependent barren plateaus in shallow quantum neural networks. arXiv preprint ArXiv:2001.00550, 2020 .

[14] K. Sharma, M. Cerezo, L. Cincio, and P. J. Coles, Trainability of dissipative perceptron-based quantum neural networks. arXiv preprint ArXiv:2005.12458, 2020.

[15] S. Wang, E. Fontana, M. Cerezo, K. Sharma, A. Sone, L. Cincio, and P. J. Coles, Noise-induced barren plateaus in variational quantum algorithms. arXiv preprint ArXiv:2007.14384, 2020.
[16] Z. Holmes, A. Arrasmith, B. Yan, P. J. Coles, A. Albrecht, and A. T. Sornborger, Barren plateaus preclude learning scramblers. arXiv preprint ArXiv:2009.14808, 2020.

[17] M. J. Bremner, C. Mora, and A. Winter, Are Random Pure States Useful for Quantum Computation?, Phys. Rev. Lett. 102, 190502 (2009).

[18] D. Gross, S. T. Flammia, and J. Eisert, Most Quantum States Are Too Entangled To Be Useful As Computational Resources, Phys. Rev. Lett. 102, 190501 (2009).

[19] M. Kieferová and N. Wiebe, Tomography and generative training with quantum Boltzmann machines, Phys. Rev. A. 96, 062327 (2017).

[20] S. Popescu, A. J. Short, and A. Winter, Entanglement and the foundations of statistical mechanics, Nat. Phys. 2, 754 (2006)

[21] R. A. Low, Large deviation bounds for $k$-designs, Proc. R. Soc. A: Math., Phys. Eng. Sci. 465, 3289 (2009).

[22] S. Goldstein, J. L. Lebowitz, R. Tumulka, and N. Zanghì, Canonical Typicality, Phys. Rev. Lett. 96, 050403 (2006).

[23] A. Sugita, On the basis of quantum statistical mechanics, Nonlinear Phenomena in Complex Syst. 10, 192 (2007).

[24] M. H. Amin, E. Andriyash, J. Rolfe, B. Kulchytskyy, and R. Melko, Quantum Boltzmann Machine, Phys. Rev. X 8, 021050 (2018).

[25] A. W. Harrow and R. A. Low, Random quantum circuits are approximate 2-designs, Commun. Math. Phys. 291, 257 (2009).

[26] F. G. S. L. Brandao, A. W. Harrow, and M. Horodecki, Local random quantum circuits are approximate polynomialdesigns, Commun. Math. Phys. 346, 397 (2016).

[27] J. Haferkamp, D. Hangleiter, A. Bouland, B. Fefferman, J. Eisert, and J. Bermejo-Vega, Closing gaps of a quantum advantage with short-time Hamiltonian dynamics. arXiv preprint ArXiv:1908.08069, 2019.

[28] Y. Nakata, C. Hirche, C. Morgan, and A. Winter, Unitary 2designs from random $x$ - and $z$-diagonal unitaries, J. Math. Phys. 58, 052203 (2017).

[29] R. Cleve, D. Leung, L. Liu, and C. Wang, Near-linear constructions of exact unitary 2-designs. arXiv preprint ArXiv:1501.04592, 2015.

[30] C. Dankert, R. Cleve, J. Emerson, and E. Livine, Exact and approximate unitary 2-designs and their application to fidelity estimation, Phys. Rev. A. 80, 012304 (2009).

[31] F. Haake, in Quantum Coherence in Mesoscopic Systems, p. 583. Springer, 1991.

[32] S. Das Sarma, D.-L. Deng, and L.-M. Duan, Machine learning meets quantum physics. arXiv preprint ArXiv:1903. 03516, 2019.

[33] J. Eisert, M. Cramer, and M. B. Plenio, Colloquium: Area laws for the entanglement entropy, Rev. Mod. Phys. 82, 277 (2010).

[34] M. B. Hastings and T. Koma, Spectral gap and exponential decay of correlations, Commun. Math. Phys. 265, 781 (2006).

[35] M. B. Hastings, An area law for one-dimensional quantum systems, J. Stat. Mech.: Theory and Exp. 2007, P08024 (2007).

[36] B. Yan, L. Cincio, and W. H. Zurek, Information Scrambling and Loschmidt Echo, Phys. Rev. Lett. 124, 160603 (2020). 
[37] The work of McClean et al. [12] can also be seen to implicitly imply barren plateaus for gradient-free methods.

[38] R. Babbush, J. McClean, D. Wecker, A. Aspuru-Guzik, and N. Wiebe, Chemical basis of Trotter-Suzuki errors in quantum chemistry simulation, Phys. Rev. A 91, 022311 (2015).
[39] E. Grant, L. Wossnig, M. Ostaszewski, and M. Benedetti, An initialization strategy for addressing barren plateaus in parametrized quantum circuits, Quantum 3, 214 (2019).

[40] N. Wiebe, Key questions for the quantum machine learner to ask themselves, New J. Phys. 22, 091001 (2020). 